\title{
Human Coronavirus NL63 in Hospitalized Children With Respiratory Infection: A 2-Year Study From Chongqing, China
}

\author{
*Chen Xin, *Zhang Zhi Yong, "Li Yan, ${ }^{*}$ Zhao Xiao Dong \\ ${ }^{*}$ Division of Nephrology and Immunology, Children’s Hospital of Chongqing Medical University, Chongqing, China; and ${ }^{\dagger}$ National \\ Microbiology Laboratory, Public Health Agency of Canada, Winnipeg, Manitoba, Canada.
}

Correspondence to: Dr Zhao Xiao Dong, Division of Nephrology and Immunology, Children's Hospital of Chongqing Medical University, Chongqing 400014, China. zhaoxd530@yahoo.com.cn. Received: January 13, 2012; Initial review: February 06, 2012; Accepted: April 10, 2012.

\begin{abstract}
Human coronavirus (HCoV) NL63, a newly discovered coronavirus, has been associated with acute lower respiratory tract infections (ALRTI). We detected HCoV-NL63 via reverse transcriptional PCR (RT-PCR) in eight out of 878 respiratory specimens freshly collected from hospitalized children with ALRTI between April 2006 and March 2008 in Children's Hospital of Chongqing Medical University. Peak of HCoV-NL63 activity often appeared during the summer and autumn in Chongqing area. All children with HCoV-NL63 infection were $<1$ year of age. The diagnosis included bronchial pneumonia, bronchitis, interstitial pneumonia and bronchiolitis. All children recovered.
\end{abstract}

Key words: Acute lower respiratory tract infections, Epidemiology, HCoV-NL63, Respiratory tract virus.

Published online: June 10, 2012. PII: S09747551200045 - 2

$\mathrm{C}$ oronavirus, a genus of the Coronaviridae family, is an enveloped virus with a large plusstrand RNA genome [1]. Human coronavirus $(\mathrm{HCoV}) 229 \mathrm{E}$ and OC43 are members of group I and II, respectively. HCoV infection is thought to be responsible for up to $30 \%$ of common cold cases in winter and occasionally causes acute lower respiratory tract disease in susceptible infants, elderly individuals, and immunocompromized adults [2-3]. In 2003, the outbreak of severe acute respiratory syndrome (SARS) led to the preliminary identification of SARScoronavirus, [4] which is currently considered to be a distinct member of the group 2 coronaviruses [5] or the first member of group 4 coronaviruses. In 2004, a new HCoV-NL63, was identified in clinical specimens from both infants and adults with acute respiratory tract infection (ARTI) in the Netherlands [1]. Sequence analysis of the complete genome of HCoV-NL63 revealed that the virus was more closely related to $\mathrm{HCoV}$ 229E than to the other human coronaviruses [1]. Preliminary data suggested that HCoV-NL63 might be an important respiratory tract pathogen in children, similarly to respiratory syncytial virus (RSV) and human metapneumovirus (hMPV) [1,6-9]. The epidemilogy and clinical features of HCoV-NL63 infection are largely unknown. We prospectively detected the presence of HCoV-NL63 in children with acute lower respiratory tract infections (ALRTI) for two consecutive years.

\section{Methods}

All children hospitalized with a diagnosis of ALRTI, between April 2006 to March 2008 were enrolled. Nasopharyngeal aspirates (NPA) were collected following informed consent, on the day of admission according to a standard protocol and transported to the virology diagnostic laboratory within 4h. The supernatants and cells of NPA were separated by centrifugation [10] and the cell-free fluid was stored in aliquots at $-80^{\circ} \mathrm{C}$ until RNA extraction. Total RNA was extracted by using the QIAamp viral RNA mini kit (Qiagen, Germany) and complementary DNA (cDNA) was synthesized by using the PrimeScript RT Reagent Kit (TaKaRa, China). Primers used for HCoV-NL63 gene amplification have been previously described $[1,11]$. The bulk PCR products were purified using a QIAquick Gel Extraction kit (Qiagen, Germany). Sequence analyses of the PCR products were performed on an ABI 3100 sequencer at core facility of nucleotide sequencing of Chongqing Medical University. The sequenced fragments of the HCoV-NL63 1a gene were assembled and analyzed with the SEQMAN, EDITSEQ, and MEGALIGN programs in the Lasergene program (DNASTAR, Madison, WI). Phylogenetic trees were generated by the neighbor-joining method using the MEGA4 program.

Co-infection with other viruses. RSV and hMPV in 
specimens were detected by traditional PCR and Realtime PCR, respectively. Positive samples for $\mathrm{HCoV}$ NL63 were also subjected to direct immunofluorescence (DFA) (Diagnostic Hybrids, America) testing for common respiratory viruses, including influenza $A$ and $B$ viruses, para-influenza virus types 1-3, and adenovirus.

Clinical evaluation and statistical analysis. The clinical diagnosis of ALRTI was based on the presence of cough, tachypnea, chest indrawing, or wheeze of $<7$ days duration, as per WHO standard protocol [12]. The medical files of HCoV-NL63 infected children were written by pediatricians. In brief, the lists included date of collection of NPA, demographic data (age, gender, hospital days, history of wheezing of patients or their families), symptoms (fever, cough, wheezing, tachypnea, cyanosis, rhinitis, hoarseness of voice, vomiting and diarrhea), laboratory testing (coinfection with viruses, white blood cell count and differential count, C-reactive protein, chest radiograph), discharge diagnosis, and outcome (amelioration or recovery).

The study was approved by Children's Hospital of Chongqing Medical University.

\section{Results}

Sample collection. During the study period, a total of 878 NPA from hospitalized children (592 males, 286 females) were collected, accounting for $13.9 \%(878 / 6306)$ of the total admissions with ALRTI during the same. The age of the patients ranged was ranging from 29 days to 15.9 years. As a result of unequal seasonal distribution of patients with respiratory diseases, $44.4 \%$ of the total samples were collected in winter (December to February); 19.1\% from March to May, 12.7\% from June to August, and $23.8 \%$ from September to November, respectively.

Clinical features. All NPA were tested for HCoV-NL63 by RT-PCR, and 8 out of 878 yielded positive results, (Table I). Patients with HCoV-NL63 aged from 1-8 mo, and the age of 6 positive patients was $<6$ months (median, 4.6 months). RSV was detected positive by RT-PCR in 3 children with HCoV-NL63 infection and hMPV positive in 2 patients. None of the patients were detected positive for other respiratory viruses. All HCoV-NL63 positive patients did not have leucocytosis in peripheral blood tests, and none of patients presented with leukopenia and lymphopenia. The concentration of $\mathrm{C}$-reactive protein was not elevated in 5 of $8 \mathrm{HCoV}-\mathrm{NL} 63$ patients tested for same. None of the 8 patients had predisposing factors or underlying diseases, nor did they require intensive care. Chest radiographs were done in 6 out of 8 children, all of them revealed varying degree of bilateral infiltrates.
Uneventful recovery or amelioration were achieved in all patients.

Phylogenetic analysis. Nucleotide sequences were analyzed for nucleotides 5778 to 6616 of the 1a gene in these 8 positive specimens. Sequence comparison with published Netherlands, Australia, Belgium, Canada, South Korea, Sweden, South Africa and Italy isolates showed that the 1a genes were relatively conserved, with nucleic acid identities ranging from $95.3 \%$ to $100 \%$ among specimens. The phylogenetic tree of the HCoVNL63 isolates showed the existence of two major groups or clusters, with the majority of isolates clustering in cluster 1 . In this study, four HCoV-NL63 isolates were found in cluster 1 , and the other four in cluster 2 .

\section{Discussion}

In the study period, 8 out of 878 children with ALRTI tested positive for HCoV-NL63, indicating a obviously low prevalence of this virus as compared to other common respiratory viruses such as RSV, hMPV, influenza $A$ and B viruses, para-influenza virus types 1-3, and adenovirus in Southwest China; it was also lower than the reported prevalence from other countries and regions $(1.1 \% \sim 7 \%)[6,7,9,13-16]$.

Surprisingly, seasonality of HCoV-NL63 was quite different from other respiratory viruses such as RSV, which showed invariable peak in the winter months in Chongqing. Some of the HCoV-NL63-positive cases; however, were identified in hot and wet summer months in this region, indicating further that long-term surveillance studies are needed to clarify epidemiological picture of HCoV-NL63. In this study, all HCoV- NL63 positive patients were under 12 months old, and most of HCoV-NL63 positive patients (6/8) were under 6 months old. These results show that HCoV-NL63 causes ALRTI in younger children, especially in infants, as shown in previous studies $[8,14]$.

In the present study, the clinical symptoms associated with HCoV-NL63 were similar to those reported previously and comparable to those observed in other viral respiratory infections. Unlike previous reports that indicated close association of HCoV-NL63 infection and croup, $[17,18]$ none of our patients presented with croup. In previous studies, $[8,9,11,15]$ some of the children with HCoV-NL63 infection experienced underlying medical problems, especially prematurity and pulmonary and cardiac diseases, whereas none of our patients experienced underlying diseases identified. None of the 8 patients required intensive care, indicating that $\mathrm{HCoV}-$ NL63 infection does not lead to relatively severe illness requiring internsive care even in infants. Unfortunately, 


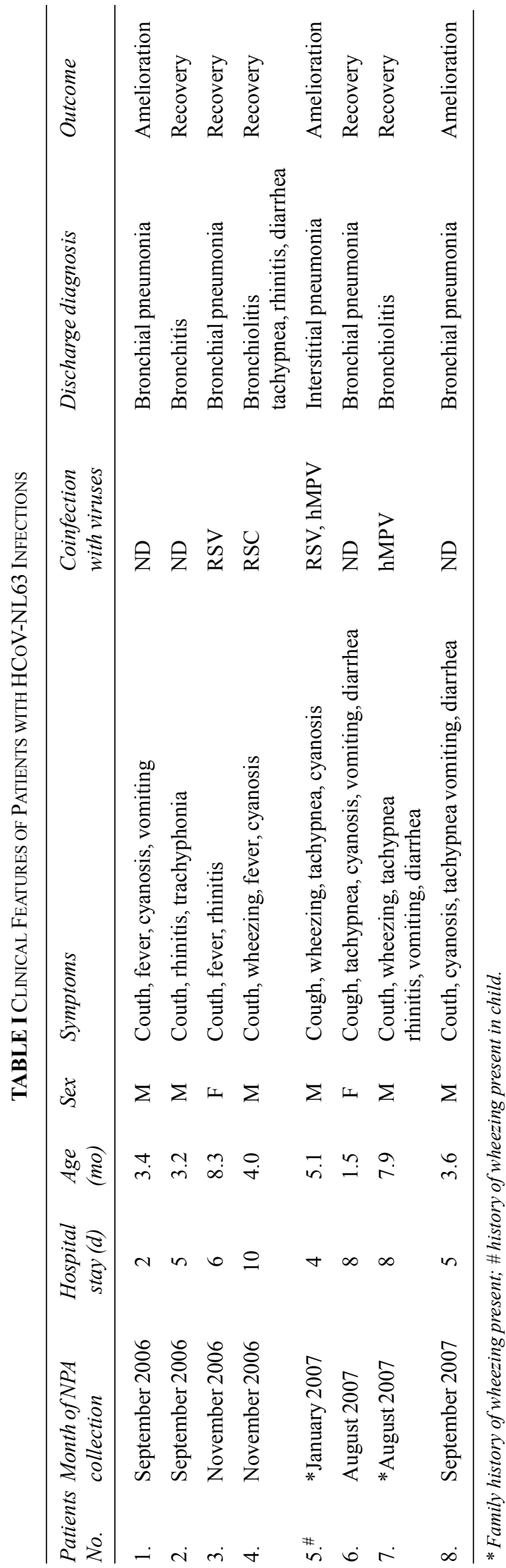

the high frequency of co-infections in HCoV-NL63 positive samples made it difficult to define the clinical character of HCoV-NL63 infection.

The phylogenetic analysis of the HCoV-NL63 isolates indicated they belonged to the separated circulation of cluster 1 viruses from September 2006 to December 2006, and cluster 2 viruses from January 2007 to September 2007. A one-year study from Canada also found all circulating isolates were in cluster 1 [11], yielding a speculation that viruses from one cluster may shift to another over time and therefore lead to periodic circulation of two cluster viruses.

In conclusion, we confirmed the presence of $\mathrm{HCoV}$ NL63 in consecutive hospitalized children with ALRTI. However, the association of this virus infection and respiratory tract illnesses remains indistinct. Therefore, population-based, prospective and multicenter study may be required to illustrate the epidemiologic picture of HCoV-NL63 in China.

Acknowledgments: Professor Yan Li (National Microbiology Laboratory, Canadian Science Center for Human and Animal Health) for thoughtful and critical review of the manuscript, and Mr. Qianglin Duan for critical reading of the manuscript. Contributors: CX: was responsible for planning and conducting experiments, data analysis, and wrote the manuscript; ZZY: took part in the collection of samples, RNA extraction, cDNA synthesis and detection co-infection with RSV. LY provided the sequence of primers used for HCoV-NL63 gene amplification and gave some thoughtful and critical reviews of the manuscript and ZXD: has designed research, provided project guidance, suggested experimental design, reviewed data analysis and interpretations, and critically revised the manuscript.

Funding: The National Natural Science Foundation of China (No.30730098) and Natural Science Foundation Project of CQ CSTC (No.cstc2011jjA10083).

Competing interests: None stated.

\section{REFERENCES}

1. van der Hoek L, Pyrc K, Jebbink MF, Vermeulen-Oost W, Berkhout RJ, Wolthers KC, et al. Identification of a new human coronavirus. Nat Med. 2004;10:368-73.

2. Falsey AR, Walsh EE, Hayden FG. Rhinovirus and coronavirus infection- associated hospitalizations among older adults. J Infect Dis. 2002;185:1338-41.

3. Pene F, Merlat A, Vabret A, Rozenberg F, Buzyn A, Dreyfus F, et al. Coronavirus 229E-related pneumonia in immunocompromised patients. Clin Infect Dis. 2003;37:929-32.

4. Kuiken T, Fouchier RA, Schutten M, Rimmelzwaan GF, van Amerongen G, van Riel D, et al. Newly discovered coronavirus as the primary cause of severe acute respiratory syndrome. Lancet. 2003;362:263-70.

5. Snijder EJ, Bredenbeek PJ, Dobbe JC, Thiel V, Ziebuhr J, Poon LL, et al. Unique and conserved features of genome and proteome of SARS-coronavirus, an early split-off 
from the coronavirus group 2 lineage. J Mol Biol. 2003;331:991-1004.

6. Bastien N, Anderson K, Hart L, Van Caeseele P, Brandt $\mathrm{K}$, Milley D, et al. Human coronavirus NL63 infection in Canada. J Infect Dis. 2005;191:503-6.

7. Chiu SS, Chan KH, Chu KW, Kwan SW, Guan Y, Poon LL, et al. Human coronavirus NL63 infection and other coronavirus infections in children hospitalized with acute respiratory disease in Hong Kong, China. Clin Infect Dis. 2005;40:1721-9.

8. Esper F, Weibel C, Ferguson D, Landry ML, Kahn JS. Evidence of a novel human coronavirus that is associated with respiratory tract disease in infants and young children. J Infect Dis. 2005;191:492-8.

9. Moës E, Vijgen L, Keyaerts E, Zlateva K, Li S, Maes P, et al. A novel pancoronavirus RT-PCR assay: frequent detection of human coronavirus NL63 in children hospitalized with respiratory tract infections in Belgium. BMC Infect Dis. 2005;5:6.

10. Maggi F, Pifferi M, Vatteroni M, Fornai C, Tempestini E, Anzilotti S, et al. Human metapneumovirus associated with respiratory tract infections in a 3-year study of nasal swabs from infants in Italy. $\mathrm{J}$ Clin Microbiol. 2003;41:2987-91.

11. Bastien N, Robinson JL, Tse A, Lee BE, Hart L, Li Y. Human Coronavirus NL-63 Infections in Children: a 1-
Year Study. J Clin Microbiol. 2005;439:4567-73.

12. Cuevas LE, Nasser AM, Dove W, Gurgel RQ, Greensill J, Hart CA. Human metapneumovirus and respiratory syncytial virus, Brazil. Emerg Infect Dis 2003;9:1626-8.

13. Arden KE, Nissen MD, Sloots TP, Mackay IM. New human coronavirus, HCoV-NL63, associated with severe lower respiratory tract disease in Australia. J Med Virol. 2005;75:455-62.

14. Vabret A, Mourez T, Dina J, van der Hoek L, Gouarin S, Petitjean J, et al. Human coronavirus NL63, France. Emerg Infect Dis. 2005;11:1225-9.

15. Fouchier RA, Hartwig NG, Bestebroer TM, Niemeyer B, de Jong JC, Simon JH, et al. A previously undescribed coronavirus associated with respiratory disease in humans. Proc Natl Acad Sci U S A. 2004;101:6212-6.

16. Kaiser L, Regamey N, Roiha H, Deffernez C, Frey U. Human coronavirus NL63 associated with lower respiratory tract symptoms in early life. Pediatr Infect Dis J. 2005;24:1015-7.

17. van der Hoek L, Sure K, Ihorst G, Stang A, Pyrc K, Jebbink MF, et al. Croup Is Associated with the Novel Coronavirus NL63. PLoS Med. 2005;2:e240.

18. Leung TF, Li CY, Lam WY, Wong GW, Cheuk E, Ip M, et al. Epidemiology and clinical presentations of human coronavirus NL63 infections in hong kong children. J Clin Microbiol. 2009;47: 3486-92. 